\title{
Remark about non-relativistic string in Newton-Cartan background and null reduction
}

\section{J. Klusoň}

Department of Theoretical Physics and Astrophysics, Faculty of Science, Masaryk University,

Kotlářská 2, 611 37, Brno, Czech Republic

E-mail: klu@physics.muni.cz

ABStract: We analyze non-relativistic string in Newton-Cartan background that was found recently in arXiv:1705.03535. We find its Hamiltonian formulation and study structure of constraints. We also discuss a relation between string in Newton-Cartan Background and T-duality along null reduction.

KeYwords: Bosonic Strings, AdS-CFT Correspondence

ArXiv EPrint: 1803.07336 


\section{Contents}

1 Introduction 1

2 Review of non-relativistic string in Newton-Cartan background 2

3 Canonical analysis $\quad 4$

3.1 Algebra of constraints 5

$\begin{array}{ll}3.2 & \text { Fixing gauge }\end{array}$

$\begin{array}{lll}3.3 & \text { Scaling limit } & 7\end{array}$

4 Alternative derivation of non-relativistic string $\quad 8$

5 Conclusion

\section{Introduction}

Today it is well known that non-relativistic holography is a very useful for description of strongly coupled condensed matter systems, see for example [1]. In fact, non-relativistic holography is an example of non-Lorentzian systems that have been studying last few years very intensively. For example, effective actions of non-relativistic field theories were analyzed in [2-6]. Further, non-relativistic local symmetries are crucial for the proposal of renormalizable theory of gravity known today as Hořava-Lifshitz gravity [7]. In fact, recently close relation between Hořava-Lifshitz gravity and Newton-Cartan gravity was found in [8]. Finally, three dimensional non-relativistic gravities were also very intensively studied in $[9,10]$.

Certainly it is very interesting question to analyze extended object in the context of non-relativistic gravity. Non-relativistic strings were firstly introduced from different point of view in $[11,12]$, for further analysis in the context of string theory, see for example [1317]. Non-relativistic strings and p-branes gained renewed interest recently when these objects were studied in the context of Newton-Cartan gravity and Carroll gravity [18-33].

There are basically two ways how to derive non-relativistic string in Newton-Cartan background. The first one is based on the gauging procedure of non-relativistic string in the flat background which was introduced in [21]. The characteristic property of this approach is that the number of longitudinal dimensions is doubled with respect to standard NewtonCartan gravity. This fact also naturally emerges when we construct non-relativistic strings or p-branes in Newton-Cartan background implementing the limiting procedure [34, 35]. However there is an alternative procedure how to define Newton-Cartan theory which is based upon null dimensional reduction [36-38] of higher dimensional theory. Then one can ask the question whether null dimensional reduction of the Polyakov action leads to new action for the string in Newton-Cartan background. This question was answered in a very 
nice paper [27] where new covariant action for string in Newton-Cartan background was found. It was further shown there that performing the second non-relativistic limit that affects both target space and world-sheet coordinates leads to sigma models that describe strings moving in novel non-Lorentzian geometry. The main difference between this approach and the construction of non-relativistic string performed in [21] is that we obtain string moving in Newton-Cartan gravity without doubling the number of longitudinal directions. This is a very attractive property of this construction. However the price that we have to pay for it is that now there is an additional mode that propagates on the world-volume of the non-relativistic string. The presence of this mode is a reflection of the fact that non-relativistic string in Newton-Cartan background is constructed through dimensional reduction from higher dimensional space-time with null isometry.

Since this proposal is very interesting it deserves further study. The goal of this paper is precisely focused on this analysis. In the first part we determine Hamiltonian form of the action introduced in [27]. We find this Hamiltonian as the sum of two constraints which are manifestly invariant under Galilean transformations. We further show that these constraints are the first class constraints with agreement with the fact that the string action is invariant under world-sheet diffeomorphism.

At the second part of this paper we focus on alternative derivation of the non-relativistic string in Newton-Cartan background that reflects its deep string theory origin. In more details, we start with the Hamiltonian formulation of the string in the background with null isometry. Then we show that when we study string in this background the correct way how to perform dimensional reduction in case of the fundamental string is to perform T-duality along this direction. It is well known that string T-duality can be interpreted as canonical transformation [39, 40]. Performing this canonical transformation for the string in the background with null isometry we find that the Hamiltonian constraint takes exactly the same form as the Hamiltonian constraint for the string in Newton-Cartan background that we found in the first section. Finally we perform an inverse Legendre transformation and we find that resulting Lagrangian density exactly coincides with the Lagrangian found in [27] which is again nice consistency check.

This paper is organized as follows. In the next section 2 we review the construction of non-relativistic string in Newton-Cartan background as was performed in [27]. Then in section 3 we perform canonical analysis of this theory, determine constraint structure and calculate Poisson algebra of constraints. We also discuss second non-relativistic limit in the context of Hamiltonian formulation. We also briefly discuss the gauge fixed theory. In section 4 we show that non-relativistic string in Newton-Cartan background can be defined starting with the string in the background with null isometry and then performing T-duality along null direction. Finally in conclusion 5 we outline our result and suggest possible extension of this work.

\section{Review of non-relativistic string in Newton-Cartan background}

In this section we review the construction of non-relativistic string in Newton-Cartan background as was presented [27]. The starting point is the Polyakov action in general back- 
ground

$$
S=\int d^{2} \sigma \mathcal{L}=-\frac{T}{2} \int d^{2} \sigma \sqrt{-\gamma} \gamma^{\alpha \beta} g_{\alpha \beta}, \quad g_{\alpha \beta}=\partial_{\alpha} x^{M} \partial_{\beta} x^{N} G_{M N},
$$

where $G_{M N}$ is $d+2$ dimensional target space time metric, $x^{M}, M, N=0, \ldots, d+1$ determine position of the string in the target space-time and $T$ is the string tension. Finally, $\gamma_{\alpha \beta}$ is two dimensional world-sheet metric where $\gamma=\operatorname{det} \gamma_{\alpha \beta}, \gamma_{\alpha \beta} \gamma^{\beta \delta}=\delta_{\alpha}^{\delta}$, where $\alpha=0,1$ and where we label world-sheet metric with coordinates $\sigma^{\alpha}$ so that $\partial_{\alpha} \equiv \frac{\partial}{\partial \sigma^{\alpha}}$.

As the next step we consider the target space metric in the form

$$
d s^{2}=G_{M N} d x^{M} d x^{N}=2 \tau(d u-m)+h_{\mu \nu} d x^{\mu} d x^{\nu},
$$

where $\mu, \nu=0,1, \ldots, d, M=(u, \mu)$ and where

$$
\tau=\tau_{\mu} d x^{\mu}, \quad m=m_{\mu} d x^{\mu},
$$

where $\operatorname{det} h_{\mu \nu}=0$. The tensors $\tau_{\mu}, m_{\mu}$ and $h_{\mu \nu}$ are independent of $u$. We also define $e_{\mu}^{a}$ through the relation

$$
h_{\mu \nu}=e_{\mu}^{a} e_{\nu}^{b} \delta_{a b}, \quad a=1, \ldots, d .
$$

Now we are ready to proceed to the analysis introduced in [27]. The main idea is to remove the field $x^{u}$ from the description. First of all we define momentum current conjugate to $u$ as

$$
P_{u}^{\alpha}=\frac{\partial \mathcal{L}}{\partial\left(\partial_{\alpha} x^{u}\right)}=-T \sqrt{-\gamma} \gamma^{\alpha \beta} G_{u \mu} \partial_{\beta} x^{\mu}=-T \sqrt{-\gamma} \gamma^{\alpha \beta} \tau_{\beta},
$$

where $\tau_{\alpha}=\tau_{\mu} \partial_{\alpha} x^{\mu}$. Note that the equation of motion for $x^{u}$ has the form

$$
\partial_{\alpha}\left(\frac{\partial \mathcal{L}}{\partial_{\alpha} x^{u}}\right)=\partial_{\alpha} P_{u}^{\alpha}=0
$$

so that the condition of imposing $x^{u}$ on-shell is equivalent to the condition

$$
\partial_{\alpha} P_{u}^{\alpha}=0 \text {. }
$$

To proceed further we require that $P_{u}^{\alpha}$ is an independent variable which can be imposed by the Legendre transformations

$$
\hat{\mathcal{L}}=\mathcal{L}-P_{u}^{\alpha} \partial_{\alpha} x^{u}
$$

where $\hat{\mathcal{L}}$ is independent on $x^{u}$. On the other hand imposing $P_{u}^{\alpha}$ to be on shell implies relation between metric components $\gamma^{\alpha \beta}$. We will solve $\gamma^{\alpha \beta}$ using $P_{u}^{\alpha}$ and $\tau_{\alpha}$ as follows. The solution of this equation can be written as [27]

$$
\sqrt{-\gamma} \gamma^{\alpha \beta}=e\left(-v^{\alpha} v^{\beta}+e^{\alpha} e^{\beta}\right)
$$

where

$$
e_{\alpha}=\frac{\epsilon_{\alpha \beta} P_{u}^{\beta}}{T}, \quad v^{\alpha}=-\frac{P_{u}^{\alpha}}{P_{u}^{\gamma} \tau_{\gamma}}, \quad e^{\alpha}=-T \frac{\epsilon^{\alpha \beta} \tau_{\beta}}{P_{u}^{\gamma} \tau_{\gamma}},
$$


and where

$$
e=\operatorname{det}\left(\begin{array}{cc}
\tau_{\tau} & e_{\tau} \\
\tau_{\sigma} & e_{\sigma}
\end{array}\right)=\tau_{\tau} e_{\sigma}-\tau_{\sigma} e_{\tau}=\epsilon^{\alpha \beta} \tau_{\alpha} e_{\beta},
$$

where we also defined $\epsilon^{\tau \sigma}=-\epsilon_{\tau \sigma}=1$ so that $\epsilon^{\alpha \gamma} \epsilon_{\gamma \beta}=\delta_{\beta}^{\alpha}$. Using this result we can write the Lagrangian density $\hat{\mathcal{L}}$ as

$$
\hat{\mathcal{L}}=-\frac{T}{2} \sqrt{-\gamma} \gamma^{\alpha \beta} \hat{h}_{\alpha \beta}=-\frac{T}{2} e\left(-v^{\alpha} v^{\beta}+e^{\alpha} e^{\beta}\right) \hat{h}_{\alpha \beta},
$$

where $\hat{h}_{\mu \nu}=h_{\mu \nu}-m_{\mu} \tau_{\nu}-\tau_{\mu} m_{\nu}, \hat{h}_{\alpha \beta}=\hat{h}_{\mu \nu} \partial_{\alpha} x^{\mu} \partial_{\beta} x^{\nu}$.

As the final step we solve the equation of motion (2.7). This equation can be solved locally by $e_{\alpha}=\partial_{\alpha} \eta$ and we substitute this result into the action $S=\int d^{2} \sigma \hat{\mathcal{L}}$. Then we obtain following Lagrangian $\hat{\mathcal{L}}$ in the form

$$
\begin{aligned}
\hat{\mathcal{L}}= & -T \epsilon^{\alpha \beta} \partial_{\beta} \eta m_{\mu} \partial_{\alpha} x^{\mu}+ \\
& +\frac{T}{2} \frac{\epsilon^{\alpha \alpha^{\prime}} \epsilon^{\beta \beta^{\prime}}\left(\partial_{\alpha^{\prime}} \eta \partial_{\beta^{\prime}} \eta-\tau_{\mu} \partial_{\alpha^{\prime}} x^{\mu} \tau_{\nu} \partial_{\beta^{\prime}} x^{\nu}\right)}{\epsilon^{\gamma \gamma^{\prime}} \tau_{\mu} \partial_{\gamma} x^{\mu} \partial_{\gamma^{\prime}} \eta} h_{\mu \nu} \partial_{\alpha} x^{\mu} \partial_{\beta} x^{\nu} .
\end{aligned}
$$

This Lagrangian density is the starting point for the canonical analysis.

\section{Canonical analysis}

In this section we perform canonical analysis of the Lagrangian density (2.13). First of all we derive following conjugate momenta

$$
p_{\eta}=\frac{\partial \hat{\mathcal{L}}}{\partial\left(\partial_{0} \eta\right)}=T m_{\sigma}-T \frac{\epsilon^{\beta \beta^{\prime}} \partial_{\beta^{\prime}} \eta}{\epsilon^{\gamma \gamma^{\prime}} \tau_{\gamma} \partial_{\gamma^{\prime}} \eta} h_{\sigma \beta}+\frac{T}{2} \frac{\epsilon^{\alpha \alpha^{\prime}} \epsilon^{\beta \beta^{\prime}}\left(\partial_{\alpha^{\prime}} \eta \partial_{\beta^{\prime}} \eta-\tau_{\alpha^{\prime}} \tau_{\beta^{\prime}}\right)}{\left(\epsilon^{\gamma \gamma^{\prime}} \tau_{\gamma} \partial_{\gamma^{\prime}} \eta\right)^{2}} h_{\alpha \beta} \tau_{\sigma}
$$

and

$$
\begin{aligned}
p_{\mu}= & \frac{\partial \hat{\mathcal{L}}}{\partial\left(\partial_{0} x^{\mu}\right)}=-T \partial_{\sigma} \eta m_{\mu}+T \frac{\epsilon^{\beta \beta^{\prime}}\left(\partial_{\sigma} \eta \partial_{\beta^{\prime}} \eta-\tau_{\sigma} \tau_{\beta^{\prime}}\right)}{\epsilon^{\gamma \gamma^{\prime}} \tau_{\gamma} \partial_{\gamma^{\prime}} \eta} h_{\mu \nu} \partial_{\beta} x^{\nu} \\
& +T \frac{\epsilon^{\beta \beta^{\prime}} \tau_{\mu} \tau_{\beta^{\prime}}}{\epsilon^{\gamma \gamma^{\prime}} \tau_{\gamma} \partial_{\gamma^{\prime}} \eta} h_{\sigma \beta}-\frac{T}{2} \frac{\epsilon^{\alpha \alpha^{\prime}} \epsilon^{\beta \beta^{\prime}}\left(\partial_{\alpha^{\prime}} \eta \partial_{\beta^{\prime}} \eta-\tau_{\alpha^{\prime}} \tau_{\beta^{\prime}}\right)}{\left(\epsilon^{\gamma \gamma^{\prime}} \tau_{\gamma} \partial_{\gamma^{\prime}} \eta\right)^{2}} h_{\alpha \beta} \tau_{\mu} \partial_{\sigma} \eta .
\end{aligned}
$$

It is easy to see that (3.1) and (3.2) imply following primary constraint

$$
\mathcal{H}_{\sigma}=p_{\eta} \partial_{\sigma} \eta+p_{\mu} \partial_{\sigma} x^{\mu} \approx 0
$$

while we find that the bare Hamiltonian is zero

$$
H_{\text {bare }}=p_{\eta} \partial_{0} \eta+p_{\mu} \partial_{0} x^{\mu}-\hat{\mathcal{L}}=0
$$

with agreement with the fact that the action $S=\int d^{2} \sigma \hat{\mathcal{L}}$ is invariant under two dimensional diffeomorphism. On the other hand we are still missing Hamiltonian constraint. In order to find it we have to introduce following objects $e_{a}^{\mu}$ and $v^{\mu}$ that are defined as

$$
\begin{aligned}
& e_{\mu}{ }^{a} e^{\mu}{ }_{b}=\delta_{b}^{a}, \quad e_{\mu}{ }^{a} e^{\nu}{ }_{a}=\delta_{\mu}^{\nu}+\tau_{\mu} v^{\nu}, \quad v^{\mu} \tau_{\mu}=-1, \\
& v^{\mu} e_{\mu}{ }^{a}=0, \quad \tau_{\mu} e^{\mu}{ }_{a}=0 .
\end{aligned}
$$


The special and temporal vierbeins define special and temporal metrics as follows

$$
\begin{aligned}
& \tau_{\mu \nu}=\tau_{\mu} \tau_{\nu}, \quad \tau^{\mu \nu}=v^{\mu} v^{\nu}, \\
& h_{\mu \nu}=e_{\mu}{ }^{a} e_{\nu}{ }^{b} \delta_{a b}, \quad h^{\mu \nu}=e^{\mu}{ }_{a} e^{\nu}{ }_{b} \delta^{a b} .
\end{aligned}
$$

To proceed further we observe that we can write

$$
\begin{aligned}
& \frac{T^{2}}{\left(\epsilon^{\gamma \gamma^{\prime}} \tau_{\gamma} \partial_{\gamma^{\prime}} \eta\right)^{2}} \epsilon^{\beta \beta^{\prime}}\left(\partial_{\sigma} \eta \partial_{\beta^{\prime}} \eta-\tau_{\sigma} \tau_{\beta^{\prime}}\right) h_{\beta \alpha} \epsilon^{\alpha \beta^{\prime \prime}}\left(\partial_{\sigma} \eta \partial_{\beta^{\prime \prime}} \eta-\tau_{\sigma} \tau_{\beta^{\prime \prime}}\right)= \\
= & \frac{T^{2}}{\left(\epsilon^{\gamma \gamma^{\prime}} \tau_{\gamma} \partial_{\gamma^{\prime}} \eta\right)^{2}}\left(\partial_{\sigma} \eta \partial_{\sigma} \eta-\tau_{\sigma} \tau_{\sigma}\right) \epsilon^{\alpha \alpha^{\prime}} \epsilon^{\beta \beta^{\prime}}\left(\partial_{\alpha^{\prime}} \eta \partial_{\beta^{\prime}} \eta-\tau_{\alpha^{\prime}} \tau_{\beta^{\prime}}\right) h_{\alpha \beta}+T^{2} h_{\sigma \sigma}
\end{aligned}
$$

and also

$$
\begin{aligned}
& \left(p_{\mu}+T \partial_{\sigma} \eta m_{\mu}\right) v^{\mu} \partial_{\sigma} \eta-\left(p_{\eta}-T m_{\mu} \partial_{\sigma} x^{\mu}\right) \tau_{\sigma}= \\
= & \frac{T}{2\left(\epsilon^{\gamma \gamma^{\prime}} \tau_{\gamma} \partial_{\gamma^{\prime}} \eta\right)^{2}} \epsilon^{\alpha \alpha^{\prime}} \epsilon^{\beta \beta^{\prime}}\left(\partial_{\alpha^{\prime}} \eta \partial_{\beta^{\prime}} \eta-\tau_{\alpha^{\prime}} \tau_{\beta^{\prime}}\right) h_{\alpha \beta}\left(\partial_{\sigma} \eta \partial_{\sigma} \eta-\tau_{\sigma} \tau_{\sigma}\right)+T h_{\sigma \sigma} .
\end{aligned}
$$

If we combine these two relations together we obtain following primary Hamiltonian constraint

$$
\begin{aligned}
\mathcal{H}_{\tau} \equiv & \left(p_{\mu}+T \partial_{\sigma} \eta m_{\mu}\right) h^{\mu \nu}\left(p_{\nu}+T \partial_{\sigma} \eta m_{\nu}\right)- \\
& -2 T\left(p_{\mu}+T \partial_{\sigma} \eta m_{\mu}\right) v^{\mu} \partial_{\sigma} \eta+2 T\left(p_{\eta}-T m_{\mu} \partial_{\sigma} x^{\mu}\right) \tau_{\sigma}+T^{2} h_{\sigma \sigma} \approx 0 .
\end{aligned}
$$

It is instructive to rewrite this constraint into the form

$$
\mathcal{H}_{\tau}=p_{\mu} h^{\mu \nu} p_{\nu}-2 T p_{\mu} \hat{v}^{\mu} \partial_{\sigma} \eta+T^{2} \hat{h}_{\mu \nu} \partial_{\sigma} x^{\mu} \partial_{\sigma} x^{\nu}+2 T p_{\eta} \tau_{\mu} \partial_{\sigma} x^{\mu}+2 T^{2} \partial_{\sigma} \eta \Phi \partial_{\sigma} \eta,
$$

where

$$
\hat{h}_{\mu \nu}=h_{\mu \nu}-m_{\mu} \tau_{\nu}-m_{\nu} \tau_{\mu}, \quad \Phi=-m_{\mu} v^{\mu}+\frac{1}{2} m_{\mu} h^{\mu \nu} m_{\nu}, \quad \hat{v}^{\mu}=v^{\mu}-h^{\mu \nu} m_{\nu} .
$$

These objects are invariant under local Galilean transformations whose non-zero transformation rules are

$$
\delta e_{\mu}^{a}=\tau_{\mu} \lambda^{a}, \quad \delta v^{\mu}=e_{a}^{\mu} \lambda^{a}, \quad \delta m_{\mu}=e_{\mu}^{a} \lambda_{a},
$$

where $\lambda_{a}$ is parameter of local Galilean transformations.

In summary, we have found that the Hamiltonian of non-relativistic string is the sum of two primary constraints (3.3) and (3.10). In the next subsection we will analyze Poisson algebra of these constraints.

\subsection{Algebra of constraints}

As usual we have to determine an algebra of constraints. We define smeared form of these constraints as

$$
\mathbf{T}_{T}(N)=\int d \sigma N \mathcal{H}_{\tau}, \quad \mathbf{T}_{S}\left(N^{\sigma}\right)=\int d \sigma N^{\sigma} \mathcal{H}_{\sigma}
$$

so that we have

$$
\left\{\mathbf{T}_{S}\left(N^{\sigma}\right), \mathbf{T}_{S}\left(M^{\sigma}\right)\right\}=\mathbf{T}_{S}\left(N^{\sigma} \partial_{\sigma} M^{\sigma}-M^{\sigma} \partial_{\sigma} N^{\sigma}\right)
$$


To proceed further we calculate

$$
\left\{\mathbf{T}_{S}\left(N^{\sigma}\right), \mathcal{H}_{\tau}\right\}=-2 \partial_{\sigma} N^{\sigma} \mathcal{H}_{\tau}-N^{\sigma} \partial_{\sigma} \mathcal{H}_{\tau}
$$

using

$$
\begin{aligned}
& \left\{\mathbf{T}_{S}\left(N^{\sigma}\right), p_{\mu}\right\}=-\partial_{\sigma}\left(N^{\sigma} p_{\mu}\right), \quad\left\{\mathbf{T}_{S}\left(N^{\sigma}\right), x^{\mu}\right\}=-N^{\sigma} \partial_{\sigma} x^{\mu} \\
& \left\{\mathbf{T}_{S}\left(N^{\sigma}\right), p_{\eta}\right\}=-\partial_{\sigma}\left(N^{\sigma} p_{\eta}\right), \quad\left\{\mathbf{T}_{S}\left(N^{\sigma}\right), \eta\right\}=-N^{\sigma} \partial_{\sigma} \eta
\end{aligned}
$$

Then (3.15) can be equivalently written as

$$
\left\{\mathbf{T}_{S}\left(N^{\sigma}\right), \mathbf{T}_{T}(M)\right\}=\mathbf{T}_{T}\left(N^{\sigma} \partial_{\sigma} M-M \partial_{\sigma} N\right) .
$$

Finally we calculate Poisson bracket between smeared form of Hamiltonian constraints and we obtain

$$
\begin{aligned}
& \left\{\mathbf{T}_{T}(N), \mathbf{T}_{T}(M)\right\}=\int d \sigma\left(N \partial_{\sigma} M-M \partial_{\sigma} N\right) 4 T^{2} p_{\nu} h^{\nu \mu} \hat{h}_{\mu \rho} \partial_{\sigma} x^{\rho}+ \\
& +\int d \sigma\left(N \partial_{\sigma} M-M \partial_{\sigma} N\right) 4 T p_{\nu} h^{\nu \mu} \tau_{\mu} p_{\eta}- \\
& -\int d \sigma\left(N \partial_{\sigma} M-M \partial_{\sigma} N\right) 4 T^{3} \hat{v}^{\mu} \hat{h}_{\mu \nu} \partial_{\sigma} x^{\nu} \partial_{\sigma} \eta- \\
& -\int d \sigma\left(N \partial_{\sigma} M-M \partial_{\sigma} N\right) 4 T^{2} \hat{v}^{\mu} \tau_{\mu} p_{\eta} \partial_{\sigma} \eta- \\
& -\int d \sigma\left(N \partial_{\sigma} M-M \partial_{\sigma} N\right) 4 T^{2} p_{\mu} \hat{v}^{\mu} \tau_{\nu} \partial_{\sigma} x^{\nu}+ \\
& +\int d \sigma\left(N \partial_{\sigma} M-M \partial_{\sigma} N\right) 8 T^{3} \tau_{\mu} \partial_{\sigma} x^{\mu} \Phi \partial_{\sigma} \eta
\end{aligned}
$$

To proceed further we use the fact that

$$
\begin{aligned}
& h^{\mu \nu} \tau_{\nu}=0, \quad h^{\nu \mu} h_{\mu \rho}=\delta_{\rho}^{\nu}+v^{\nu} \tau_{\rho}, \\
& h^{\nu \mu} \hat{h}_{\mu \rho}=\delta_{\rho}^{\nu}+\hat{v}^{\nu} \tau_{\rho}, \quad \hat{v}^{\mu} \hat{h}_{\mu \nu}=2 \Phi \tau_{\nu} .
\end{aligned}
$$

Then if we combine these results together in (3.18) we obtain desired result

$$
\left\{\mathbf{T}_{T}(N), \mathbf{T}_{T}(M)\right\}=4 T^{2} \mathbf{T}_{S}\left(N \partial_{\sigma} M-M \partial_{\sigma} N\right) .
$$

We see that the Poisson brackets (3.14), (3.17) and (3.20) close on the constraint surface $\mathcal{H}_{\sigma} \approx 0, \mathcal{H}_{\tau} \approx 0$ and hence they are the first class constraints.

\subsection{Fixing gauge}

We have seen that the non-relativistic string Hamiltonian is the sum of two first class constraints. The natural way how to deal with such a theory is to gauge fix these constraints. For example, we can introduce following gauge fixing functions

$$
\mathcal{G}_{\tau} \equiv \sqrt{T} x^{0}-\tau \approx 0, \quad \mathcal{G}_{\sigma} \equiv \sqrt{T} \eta-\sigma \approx 0
$$


To see this that they are suitable gauge fixing functions we calculate following Poisson brackets

$$
\begin{aligned}
& \left\{\mathcal{G}_{\tau}(\sigma), \mathcal{H}_{\tau}\left(\sigma^{\prime}\right)\right\} \approx\left(2 \sqrt{T} h^{0 \nu} p_{\nu}-2 T \hat{v}^{0}\right) \delta\left(\sigma-\sigma^{\prime}\right), \quad\left\{\mathcal{G}_{\tau}(\sigma), \mathcal{H}_{\sigma}\left(\sigma^{\prime}\right)\right\} \approx 0 \\
& \left\{\mathcal{G}_{\sigma}(\sigma), \mathcal{H}_{\tau}\left(\sigma^{\prime}\right)\right\}=2 T^{3 / 2} \tau_{\mu} \partial_{\sigma} x^{\mu} \delta\left(\sigma-\sigma^{\prime}\right), \quad\left\{\mathcal{G}_{\sigma}(\sigma), \mathcal{H}_{\sigma}\left(\sigma^{\prime}\right)\right\} \approx \delta\left(\sigma-\sigma^{\prime}\right) .
\end{aligned}
$$

Since these Poisson brackets do not vanish on the constraint surface $\mathcal{H}_{\tau} \approx 0, \mathcal{H}_{\sigma} \approx 0, \mathcal{G}_{\tau} \approx$ $0, \mathcal{G}_{\sigma} \approx 0$ we see that these gauge fixing functions together with $\mathcal{H}_{\tau} \approx 0, \mathcal{H}_{\sigma} \approx 0$ are the second class constraints that vanish strongly. Then the Hamiltonian on the reduced phase space follows from the action

$$
\begin{aligned}
S & =\int d^{2} \sigma\left(p_{\mu} \partial_{0} x^{\mu}+p_{\eta} \partial_{0} \eta-\lambda^{\tau} \mathcal{H}_{\tau}-\lambda^{\sigma} \mathcal{H}_{\sigma}\right)= \\
& =\int d^{2} \sigma\left(p_{i} \partial_{\tau} x^{i}+\frac{1}{\sqrt{T}} p_{0}\right)
\end{aligned}
$$

where we used the fact that $\mathcal{H}_{\tau}=0, \mathcal{H}_{\sigma}=0$ and we also used $\mathcal{G}_{\tau}=0$ to express $x^{0}$ as $x^{0}=\frac{1}{\sqrt{T}} \tau$. Then we see from (3.23) that it is natural to identify the gauge fixed Hamiltonian density as

$$
\mathcal{H}_{\text {fixed }}=-\frac{1}{\sqrt{T}} p_{0}
$$

where $p_{0}$ can be determined from $\mathcal{H}_{\tau}=0$, at least in principle, while from $\mathcal{H}_{\sigma}=0$ we obtain $p_{\eta}$ as

$$
p_{\eta}=-\sqrt{T} p_{i} \partial_{\sigma} x^{i} .
$$

Let us consider for example a flat Newton-Cartan background when $m_{\mu}=0, \tau_{\mu}=\delta_{\mu}^{0}, h_{\mu \nu}=$ $\delta_{i j} \delta_{\mu}^{i} \delta_{\nu}^{j}$. Then clearly $h^{\mu \nu}=\delta_{i}^{\mu} \delta_{j}^{\nu} \delta^{i j}, \hat{v}^{\mu}=-\delta_{0}^{\mu}$ so that

$$
\mathcal{H}_{\tau}=p_{i} \delta^{i j} p_{j}+2 \sqrt{T} p_{0}+T^{2} \delta_{i j} \partial_{\sigma} x^{i} \partial_{\sigma} x^{j}=0
$$

that can be easily solved for $p_{0}$. As a result we obtain the Hamiltonian density on the reduced phase space in the form

$$
\mathcal{H}_{\text {fixed }}=\frac{1}{T} p_{i} \delta^{i j} p_{j}+T \delta_{i j} \partial_{\sigma} x^{i} \partial_{\sigma} x^{j} .
$$

\subsection{Scaling limit}

It was shown in [27] that the second scaling limit $T \rightarrow 0$ defines new interesting class of nonrelativistic theories. Let us now implement this idea in case of the Hamiltonian formulation of this theory. From the form of the constraint $\mathcal{H}_{\tau} \approx 0$ it is clear that the naive limit $T \rightarrow 0$ in the constraint $\mathcal{H}_{\tau} \approx 0$ leads to a trivial dynamics since $\mathcal{H}_{\tau} \rightarrow p_{\mu} h^{\mu \nu} p_{\nu} \approx 0$ for $T \rightarrow 0$. In order to resolve this problem we follow the analysis proposed in [27]. Explicitly, in order to make the Hamiltonian constraint non-trivial we have to rescale the coupling to $v^{\mu}$ too. In more details, let us write $\tau_{\mu}$ as

$$
\tau_{\mu}=N \partial_{\mu} F+\beta_{\mu}, \quad v^{\mu} \beta_{\mu}=v^{\mu} h_{\mu \nu}=0, \quad v^{\mu} \tau_{\mu}=-1 .
$$


Let us consider scaling limit

$$
F=c^{2} \tilde{F}, \quad T=\frac{\tilde{T}}{c}, \quad \eta=c \tilde{\eta}, p_{\eta}=\frac{1}{c} p_{\tilde{\eta}}, \quad c \rightarrow \infty .
$$

Further, since $v^{\mu} \tau_{\mu}=-1$ that holds for all $c$ we should rescale $v^{\mu}$ as $v^{\mu}=\frac{1}{c^{2}} \tilde{v}^{\mu}$. With the help of this prescription we find that the Hamiltonian constraint scales as

$$
\mathcal{H}_{\tau} \Rightarrow \tilde{\mathcal{H}}_{\tau}=\left(p_{\mu}+\tilde{T} \partial_{\sigma} \tilde{\eta} m_{\mu}\right) h^{\mu \nu}\left(p_{\nu}+\tilde{T} \partial_{\sigma} \tilde{\eta} m_{\nu}\right)+2 \tilde{T}\left(p_{\eta}-\tilde{T} m_{\mu} \partial_{\sigma} x^{\mu}\right) \tilde{\tau}_{\sigma} \approx 0,
$$

where $\tilde{\tau}_{\mu}=N \partial_{\mu} F$.

As a check whether our approach is correct let us start with the scaled action found in $[27]$

$$
S=-\tilde{T} \int d^{2} \sigma\left(\epsilon^{\alpha \beta} m_{\alpha} \partial_{\beta} \tilde{\eta}+\frac{\epsilon^{\alpha \alpha^{\prime}} \epsilon^{\beta \beta^{\prime}} \tilde{\tau}_{\alpha^{\prime}} \tilde{\tau}_{\beta^{\prime}}}{2 \epsilon^{\gamma \gamma^{\prime}} \tilde{\tau}_{\gamma} \partial_{\gamma^{\prime}} \tilde{\eta}} h_{\alpha \beta}\right)
$$

and determine corresponding Hamiltonian. From (3.31) we derive following conjugate momenta

$$
\begin{aligned}
p_{\tilde{\eta}}= & \frac{\partial \tilde{\mathcal{L}}}{\partial \partial_{0} \tilde{\eta}}=\tilde{T} m_{\sigma}-\tilde{T} \frac{\epsilon^{\alpha \alpha^{\prime}} \epsilon^{\beta \beta^{\prime}} \tilde{\tau}_{\alpha^{\prime}} \tilde{\tau}_{\beta^{\prime}}}{2\left(\epsilon^{\gamma \gamma^{\prime}} \tilde{\tau}_{\gamma} \partial_{\gamma^{\prime}} \tilde{\eta}\right)^{2}} h_{\alpha \beta} \tilde{\tau}_{\sigma} \\
p_{\mu}= & \frac{\partial \tilde{\mathcal{L}}}{\partial \partial_{0} x^{\mu}}=-\tilde{T} m_{\mu} \partial_{\sigma} \tilde{\eta}+\tilde{T} \frac{\epsilon^{\beta \beta^{\prime}} \tilde{\tau}_{\mu} \tilde{\tau}_{\beta^{\prime}}}{\epsilon^{\gamma \gamma^{\prime}} \tilde{\tau}_{\gamma} \partial_{\gamma^{\prime}} \tilde{\eta}} h_{\sigma \beta}+ \\
& +\frac{\epsilon^{\alpha \alpha^{\prime}} \epsilon^{\beta \beta^{\prime}} \tilde{\tau}_{\alpha^{\prime}} \tilde{\tau}_{\beta^{\prime}}}{2\left(\epsilon \gamma \gamma^{\prime} \tilde{\tau}_{\gamma} \partial_{\gamma^{\prime}} \tilde{\eta}\right)^{2}} h_{\alpha \sigma} \tilde{\tau}_{\mu} \partial_{\sigma} \tilde{\eta}-\tilde{T} \frac{\epsilon^{\beta \beta^{\prime}} \tilde{\tau}_{\sigma} \tilde{\tau}_{\beta^{\prime}}}{\epsilon^{\gamma \gamma^{\prime}} \tilde{\tau}_{\gamma} \partial_{\gamma^{\prime}} \tilde{\eta}} h_{\mu \sigma} \partial_{\sigma} x^{\sigma} .
\end{aligned}
$$

Then performing the same manipulation as in previous section we derive following Hamiltonian constraint

$$
\mathcal{H}_{\tau}=\left(p_{\mu}+\tilde{T} m_{\mu} \partial_{\sigma} \tilde{\eta}\right) h^{\mu \nu}\left(p_{\nu}+\tilde{T} m_{\nu} \partial_{\sigma} \tilde{\eta}\right)+2 \tilde{T}\left(p_{\tilde{\eta}}-\tilde{T} m_{\mu} \partial_{\sigma} x^{\mu}\right) \tilde{\tau}_{\sigma} \approx 0
$$

that coincides with (3.30).

\section{Alternative derivation of non-relativistic string}

In this section we perform an alternative derivation of the non-relativistic string in the Newton-Cartan background. The starting point of our construction is the Hamiltonian for the string in the background with null isometry. We begin with Nambu-Goto form of the string action in general background

$$
S=-T \int d^{2} \sigma \sqrt{-\operatorname{det} g_{\alpha \beta}}
$$

and find its Hamiltonian form. From (4.1) we obtain conjugate momenta

$$
p_{M}=-T G_{M N} \partial_{\alpha} x^{N} g^{\alpha \tau} \sqrt{-\operatorname{det} g} .
$$

Using this relation it is easy to find two primary constraints

$$
\mathcal{H}_{\tau}=p_{M} G^{M N} p_{N}+T^{2} G_{M N} \partial_{\sigma} x^{M} \partial_{\sigma} x^{N} \approx 0, \quad \mathcal{H}_{\sigma}=p_{M} \partial_{\sigma} x^{M} .
$$


As in section 2 we now consider background metric with null isometry

$$
d s^{2}=G_{M N} d x^{M} d x^{N}=2 \tau(d u-m)+h_{\mu \nu} d x^{\mu} d x^{\nu},
$$

where

$$
\tau=\tau_{\mu} d x^{\mu}, \quad m=m_{\mu} d x^{\mu},
$$

and where $\operatorname{det} h_{\mu \nu}=0$. It can be shown that the inverse metric $G^{M N}$ has the form

$$
G^{u u}=2 \Phi, \quad G^{u \mu}=-\hat{v}^{\mu}, \quad G^{\mu \nu}=h^{\mu \nu} .
$$

In this background the Hamiltonian and diffeomorphism constraints (4.3) have the form

$$
\begin{aligned}
& \mathcal{H}_{\tau}=2 p_{u} \Phi p_{u}-2 p_{u} \hat{v}^{\mu} p_{\mu}+p_{\mu} h^{\mu \nu} p_{\nu}+2 T^{2} \tau_{\mu} \partial_{\sigma} x^{\mu} \partial_{\sigma} u+T^{2} \hat{h}_{\mu \nu} \partial_{\sigma} x^{\mu} \partial_{\sigma} x^{\nu}, \\
& \mathcal{H}_{\sigma}=p_{u} \partial_{\sigma} u+p_{\mu} \partial_{\sigma} x^{\mu} .
\end{aligned}
$$

This is the Hamiltonian constraint for the string in the null background. Note that this background possesses an isometry

$$
u \rightarrow u+\epsilon, \quad \epsilon=\text { const } .
$$

Let us now perform canonical transformation from $u$ to $\eta[39,40]$ when we presume that the generating function has the form

$$
G=\frac{T}{2} \int d \sigma\left(u \partial_{\sigma} \eta-\partial_{\sigma} u \eta\right)
$$

Let us denote the momentum conjugate to $\eta$ as $p_{\eta}$. Then from the definition of the canonical transformation we derive following relation between momenta $p_{u}, p_{\eta}$ and spatial derivatives of $u$ and $\eta$ :

$$
p_{\eta}=-\frac{\delta G}{\delta \eta}=T \partial_{\sigma} u, \quad p_{u}=\frac{\delta G}{\delta u}=T \partial_{\sigma} \eta
$$

Now we obtain canonically dual Hamiltonian when we replace $\partial_{\sigma} u$ with $\frac{1}{T} p_{\eta}$ and $p_{u}$ with $T \partial_{\sigma} \eta$ in $\mathcal{H}_{\tau}$ and $\mathcal{H}_{\sigma}$ given above and we obtain

$$
\begin{aligned}
& \mathcal{H}_{\sigma}=p_{\eta} \partial_{\sigma} \eta+p_{\mu} \partial_{\sigma} x^{\mu} \approx 0, \\
& \mathcal{H}_{\tau}=p_{\mu} h^{\mu \nu} p_{\nu}-2 T p_{\mu} \hat{v}^{\mu} \partial_{\sigma} \eta+2 \tau_{\mu} \partial_{\sigma} x^{\mu} p_{\eta}+T^{2} \hat{h}_{\mu \nu} \partial_{\sigma} x^{\mu} \partial_{\sigma} x^{\nu}+2 T^{2} \partial_{\sigma} \eta \Phi \partial_{\sigma} \eta .
\end{aligned}
$$

which precisely coincide with the Hamiltonian constraint (3.10). It is important to stress that the canonical transformation defined by the generating function (4.9) can be interpreted as T-duality transformation along $u$-direction even if this interpretation is slightly formal due to the fact that the $u$-direction is null and hence non-compact.

Finally we check our result by derivation of the Lagrangian density from the Hamiltonian $H=\int d \sigma\left(\lambda^{\tau} \mathcal{H}_{\tau}+\lambda^{\sigma} \mathcal{H}_{\sigma}\right)$, where the constraints $\mathcal{H}_{\tau} \approx 0, \mathcal{H}_{\sigma} \approx 0$ are given in (4.11). Using this Hamiltonian we easily find

$$
\begin{aligned}
\partial_{0} x^{\mu} & =\left\{x^{\mu}, H\right\}=2 \lambda^{\tau} h^{\mu \nu} p_{\nu}-2 \lambda^{\tau} T \hat{v}^{\mu} \partial_{\sigma} \eta+\lambda^{\sigma} \partial_{\sigma} x^{\mu} \\
\partial_{0} \eta & =\{\eta, H\}=2 \lambda^{\tau} \tau_{\mu} \partial_{\sigma} x^{\mu}+\lambda^{\sigma} \partial_{\sigma} \eta
\end{aligned}
$$


and we obtain following Lagrangian density

$$
\begin{aligned}
\mathcal{L} & =p_{\mu} \partial_{0} x^{\mu}+p_{\eta} \partial_{0} \eta-\lambda^{\tau} \mathcal{H}_{\tau}-\lambda^{\sigma} \mathcal{H}_{\sigma}= \\
& =\lambda^{\tau} p_{\mu} h^{\mu \nu} p_{\nu}-\lambda^{\tau} T^{2} \hat{h}_{\mu \nu} \partial_{\sigma} x^{\mu} \partial_{\sigma} x^{\nu}-2 \lambda^{\tau} T^{2} \partial_{\sigma} \eta \Phi \partial_{\sigma} \eta
\end{aligned}
$$

To proceed further we introduce $\hat{e}_{\mu}^{a}$ as

$$
\hat{e}_{\mu}^{a}=e_{\mu}^{a}-e_{b}^{\nu} \delta^{b a} m_{\nu} \tau_{\mu}
$$

that obeys

$$
\hat{e}_{\mu}^{a} h^{\mu \nu}=\delta^{a b} e_{b}^{\nu}
$$

Using this relation we easily find

$$
p_{\mu} h^{\mu \nu} p_{\nu}=\frac{1}{4\left(\lambda^{\tau}\right)^{2}}\left(\partial_{\tau} x^{\mu}+2 T \hat{v}^{\mu} \partial_{\sigma} \eta-\lambda^{\sigma} \partial_{\sigma} x^{\mu}\right) \hat{e}_{\mu}^{a} \delta_{a b} \hat{e}_{\nu}^{b}\left(\partial_{\tau} x^{\nu}+2 T \hat{v}^{\nu} \partial_{\sigma} \eta-\lambda^{\sigma} \partial_{\sigma} x^{\nu}\right)
$$

and hence we obtain Lagrangian density in the form

$$
\begin{aligned}
\mathcal{L}= & \frac{1}{4 \lambda^{\tau}}\left(\partial_{\tau} x^{\mu}+2 T \hat{v}^{\mu} \partial_{\sigma} \eta-\lambda^{\sigma} \partial_{\sigma} x^{\mu}\right) \hat{e}_{\mu}^{a} \delta_{a b} \hat{e}_{\nu}{ }^{b}\left(\partial_{\tau} x^{\nu}+2 T \hat{v}^{\nu} \partial_{\sigma} \eta-\lambda^{\sigma} \partial_{\sigma} x^{\nu}\right)- \\
& -\lambda^{\tau} T^{2} \hat{h}_{\mu \nu} \partial_{\sigma} x^{\mu} \partial_{\sigma} x^{\nu}-2 \lambda^{\tau} T^{2} \partial_{\sigma} \eta \Phi \partial_{\sigma} \eta .
\end{aligned}
$$

This Lagrangian density can be rewritten into an equivalent form if we use the relation

$$
\hat{e}_{\mu}^{a} \delta_{a b} \hat{e}_{\nu}^{b}=\hat{h}_{\mu \nu}+2 \tau_{\mu} \Phi \tau_{\nu}
$$

so that the Lagrangian density has the form

$$
\begin{aligned}
\mathcal{L}= & \frac{1}{4 \lambda^{\tau}}\left(\hat{h}_{\tau \tau}+2 \tau_{\tau} \Phi \tau_{\tau}-2 \lambda^{\sigma} \hat{h}_{\tau \sigma}-4 \lambda^{\sigma} \tau_{\tau} \Phi \tau_{\sigma}+\right. \\
& \left.+\left(\lambda^{\sigma}\right)^{2} \hat{h}_{\sigma \sigma}+\left(\lambda^{\sigma}\right)^{2} 2 \tau_{\sigma} \Phi \tau_{\sigma}\right)-\lambda^{\tau} T^{2} \hat{h}_{\sigma \sigma}-2 \lambda^{\tau} T^{2} \partial_{\sigma} \eta \Phi \partial_{\sigma} \eta
\end{aligned}
$$

Finally we eliminate $\lambda^{\tau}, \lambda^{\sigma}$ from $\mathcal{L}$. As was argued in [33] these multipliers cannot be eliminated by their equations of motion. Instead we have to examine the equations of motion for $x^{\mu}$ and $\eta$. In fact, from the equation of motion for $x^{\mu}$ we obtain

$$
\tau_{\tau}=2 \lambda^{\tau} \partial_{\sigma} \eta+\lambda^{\sigma} \tau_{\sigma}
$$

while from the equation of motion for $\eta$ we obtain

$$
\frac{1}{2 \tau_{\sigma}}\left(\partial_{\tau} \eta-\lambda^{\sigma} \partial_{\sigma} \eta\right)=\lambda^{\tau} .
$$

Inserting this result into (4.20) we obtain

$$
\lambda^{\sigma}=\frac{\tau_{\tau \sigma}-\partial_{\tau} \eta \partial_{\sigma} \eta}{\tau_{\sigma \sigma}-\partial_{\sigma} \eta \partial_{\sigma} \eta}
$$

and hence we find that $\lambda^{\tau}$ is equal to

$$
\lambda^{\tau}=-\frac{\epsilon^{\alpha \beta} \tau_{\alpha} \partial_{\beta} \eta}{2\left(\tau_{\sigma} \tau_{\sigma}-\partial_{\sigma} \eta \partial_{\sigma} \eta\right)} .
$$


With the help of these results it is easy to find the Lagrangian density in the form

$$
\begin{aligned}
\mathcal{L}= & -\frac{1}{2 \epsilon^{\gamma \gamma^{\prime}} \tau_{\gamma} \partial_{\gamma^{\prime}} \eta}\left(\tau_{\alpha} \tau_{\beta}-\partial_{\alpha} \eta \partial_{\beta} \eta\right) \epsilon^{\alpha \alpha^{\prime}} \epsilon^{\beta \beta^{\prime}} \hat{h}_{\alpha^{\prime} \beta^{\prime}} \\
& -\frac{1}{\epsilon^{\gamma \gamma^{\prime}} \tau_{\gamma} \partial_{\gamma^{\prime}} \eta}\left(\tau_{\alpha} \tau_{\beta}-\partial_{\alpha} \eta \partial_{\beta} \eta\right) \epsilon^{\alpha \alpha^{\prime}} \epsilon^{\beta \beta^{\prime}} \tau_{\alpha^{\prime}} \tau_{\beta^{\prime}} \Phi-T^{2} \epsilon^{\alpha \beta} \tau_{\alpha} \partial_{\beta} \eta \Phi .
\end{aligned}
$$

At first sight we should say that this Lagrangian density is different from the one derived in [27]. However when we perform closer examination we find that two expressions on the second line in (4.24) cancel each other and it precisely reduces into (2.13). We mean that this is really nice consistency check.

\section{Conclusion}

Let us outline our results and suggest possible extension of this work. We analyzed nonrelativistic string theory on Newton-Cartan background which was introduced in [27]. We found its Hamiltonian form and calculated an algebra of constraints. We also discussed its gauge fixed form. We also shown an alternative way of the derivation of this theory with the help of T-duality along null direction. We mean that this is very interesting result that clearly allows natural extension of this work when we analyze Green-Schwarz superstring in the background with null isometry and perform T-duality along this direction. It would be also nice to analyze the action [27] in some specific background and try to find solutions of corresponding equations of motion. We hope to return to these problems in future.

\section{Acknowledgments}

This work was supported by the Grant Agency of the Czech Republic under the grant P201/12/G028.

Open Access. This article is distributed under the terms of the Creative Commons Attribution License (CC-BY 4.0), which permits any use, distribution and reproduction in any medium, provided the original author(s) and source are credited.

\section{References}

[1] S.A. Hartnoll, A. Lucas and S. Sachdev, Holographic quantum matter, arXiv:1612.07324 [INSPIRE].

[2] D.T. Son, Newton-Cartan Geometry and the Quantum Hall Effect, arXiv:1306.0638 [INSPIRE].

[3] M.H. Christensen, J. Hartong, N.A. Obers and B. Rollier, Torsional Newton-Cartan Geometry and Lifshitz Holography, Phys. Rev. D 89 (2014) 061901 [arXiv:1311.4794] [INSPIRE].

[4] M. Geracie, D.T. Son, C. Wu and S.-F. Wu, Spacetime Symmetries of the Quantum Hall Effect, Phys. Rev. D 91 (2015) 045030 [arXiv:1407.1252] [INSPIRE]. 
[5] K. Jensen, On the coupling of Galilean-invariant field theories to curved spacetime, arXiv: 1408.6855 [INSPIRE].

[6] J. Hartong, E. Kiritsis and N.A. Obers, Schrödinger Invariance from Lifshitz Isometries in Holography and Field Theory, Phys. Rev. D 92 (2015) 066003 [arXiv:1409.1522] [INSPIRE].

[7] P. Hořava, Quantum Gravity at a Lifshitz Point, Phys. Rev. D 79 (2009) 084008 [arXiv: 0901.3775] [INSPIRE].

[8] J. Hartong and N.A. Obers, Hořava-Lifshitz gravity from dynamical Newton-Cartan geometry, JHEP 07 (2015) 155 [arXiv: 1504.07461] [INSPIRE].

[9] E.A. Bergshoeff and J. Rosseel, Three-Dimensional Extended Bargmann Supergravity, Phys. Rev. Lett. 116 (2016) 251601 [arXiv:1604.08042] [INSPIRE].

[10] J. Hartong, Y. Lei and N.A. Obers, Nonrelativistic Chern-Simons theories and three-dimensional Hořava-Lifshitz gravity, Phys. Rev. D 94 (2016) 065027 [arXiv: 1604.08054] [INSPIRE].

[11] J. Gomis and H. Ooguri, Nonrelativistic closed string theory, J. Math. Phys. 42 (2001) 3127 [hep-th/0009181] [INSPIRE].

[12] U.H. Danielsson, A. Guijosa and M. Kruczenski, IIA/B, wound and wrapped, JHEP 10 (2000) 020 [hep-th/0009182] [INSPIRE].

[13] J. Kluson, Non-Relativistic Non-BPS Dp-brane, Nucl. Phys. B 765 (2007) 185 [hep-th/0610073] [INSPIRE].

[14] J. Gomis, F. Passerini, T. Ramirez and A. Van Proeyen, Non relativistic Dp-branes, JHEP 10 (2005) 007 [hep-th/0507135] [INSPIRE].

[15] J. Gomis, J. Gomis and K. Kamimura, Non-relativistic superstrings: A New soluble sector of $A d S_{5} \times S^{5}$, JHEP 12 (2005) 024 [hep-th/0507036] [INSPIRE].

[16] J. Gomis, K. Kamimura and P.K. Townsend, Non-relativistic superbranes, JHEP 11 (2004) 051 [hep-th/0409219] [INSPIRE].

[17] J. Brugues, T. Curtright, J. Gomis and L. Mezincescu, Non-relativistic strings and branes as non-linear realizations of Galilei groups, Phys. Lett. B 594 (2004) 227 [hep-th/0404175] [INSPIRE].

[18] E. Bergshoeff, J. Gomis and L. Parra, The Symmetries of the Carroll Superparticle, J. Phys. A 49 (2016) 185402 [arXiv: 1503.06083] [INSPIRE].

[19] E. Bergshoeff, J. Gomis, M. Kovacevic, L. Parra, J. Rosseel and T. Zojer, Nonrelativistic superparticle in a curved background, Phys. Rev. D 90 (2014) 065006 [arXiv:1406.7286] [INSPIRE].

[20] E. Bergshoeff, J. Gomis and G. Longhi, Dynamics of Carroll Particles, Class. Quant. Grav. 31 (2014) 205009 [arXiv: 1405.2264] [InSPIRE].

[21] R. Andringa, E. Bergshoeff, J. Gomis and M. de Roo, 'Stringy' Newton-Cartan Gravity, Class. Quant. Grav. 29 (2012) 235020 [arXiv:1206.5176] [INSPIRE].

[22] B. Cardona, J. Gomis and J.M. Pons, Dynamics of Carroll Strings, JHEP 07 (2016) 050 [arXiv: 1605.05483] [INSPIRE].

[23] C. Batlle, J. Gomis and D. Not, Extended Galilean symmetries of non-relativistic strings, JHEP 02 (2017) 049 [arXiv: 1611.00026] [INSPIRE]. 
[24] J. Gomis and P.K. Townsend, The Galilean Superstring, JHEP 02 (2017) 105 [arXiv: 1612.02759] [INSPIRE].

[25] J. Kluson, Carroll Limit of Non-BPS Dp-brane, JHEP 05 (2017) 108 [arXiv:1702.08685] [INSPIRE].

[26] J. Kluson, Hamiltonian Analysis of Non-Relativistic Non-BPS Dp-brane, JHEP 07 (2017) 007 [arXiv: 1704.08003] [INSPIRE].

[27] T. Harmark, J. Hartong and N.A. Obers, Nonrelativistic strings and limits of the AdS/CFT correspondence, Phys. Rev. D 96 (2017) 086019 [arXiv:1705.03535] [INSPIRE].

[28] J. Kluson, Note About Integrability of Non-Relativistic String, arXiv:1705.10951 [INSPIRE].

[29] J. Kluson, Remark About Non-Relativistic p-Brane, Eur. Phys. J. C 78 (2018) 27 [arXiv: 1707.04034] [INSPIRE].

[30] J. Kluson, Canonical Analysis of Non-Relativistic Particle and Superparticle, Eur. Phys. J. C 78 (2018) 117 [arXiv: 1709.09405] [INSPIRE].

[31] A. Barducci, R. Casalbuoni and J. Gomis, Non-relativistic Spinning Particle in a Newton-Cartan Background, JHEP 01 (2018) 002 [arXiv:1710.10970] [INSPIRE].

[32] J. Kluson, Note About Hamiltonian Formalism for Newton-Cartan String and p-Brane, arXiv: 1712.07430 [INSPIRE].

[33] J. Klusoň, Hamiltonian For String in Newton-Cartan Background, arXiv:1801.10376 [INSPIRE].

[34] E. Bergshoeff, J. Rosseel and T. Zojer, Newton-Cartan (super)gravity as a non-relativistic limit, Class. Quant. Grav. 32 (2015) 205003 [arXiv: 1505. 02095] [INSPIRE].

[35] E. Bergshoeff, J. Rosseel and T. Zojer, Non-relativistic fields from arbitrary contracting backgrounds, Class. Quant. Grav. 33 (2016) 175010 [arXiv:1512.06064] [INSPIRE].

[36] C. Duval, G. Burdet, H.P. Kunzle and M. Perrin, Bargmann Structures and Newton-cartan Theory, Phys. Rev. D 31 (1985) 1841 [InSPIRE].

[37] C. Duval, G.W. Gibbons and P. Horvathy, Celestial mechanics, conformal structures and gravitational waves, Phys. Rev. D 43 (1991) 3907 [hep-th/0512188] [INSPIRE].

[38] B. Julia and H. Nicolai, Null Killing vector dimensional reduction and Galilean geometrodynamics, Nucl. Phys. B 439 (1995) 291 [hep-th/9412002] [INSPIRE].

[39] E. Alvarez, L. Álvarez-Gaumé and Y. Lozano, A Canonical approach to duality transformations, Phys. Lett. B 336 (1994) 183 [hep-th/9406206] [INSPIRE].

[40] E. Alvarez, L. Álvarez-Gaumé and Y. Lozano, An Introduction to T duality in string theory, Nucl. Phys. Proc. Suppl. 41 (1995) 1 [hep-th/9410237] [InSPIRE]. 